\title{
Resonance tuning and broadening of bowtie nanoantennas on graphene
}

\author{
Semih Cakmakyapan ${ }^{\mathrm{a}, \mathrm{b}, *}$, Levent Sahin ${ }^{\mathrm{b}, \mathrm{c}}$, Francesco Pierini ${ }^{\mathrm{b}}$, Ekmel Ozbay ${ }^{\mathrm{a}, \mathrm{b}, \mathrm{c}}$ \\ ${ }^{a}$ Department of Physics, Bilkent University, 06800 Ankara, Turkey \\ ${ }^{\mathrm{b}}$ Nanotechnology Research Center, Bilkent University, Bilkent University, 06800 Ankara, Turkey \\ ${ }^{c}$ Department of Electrical and Electronics Engineering, Bilkent University, 06800 Ankara, Turkey
}

Received 20 January 2014; received in revised form 7 February 2014; accepted 7 February 2014

Available online 22 February 2014

\begin{abstract}
Metallic bowtie antennas are used in nanophotonics applications in order to confine the electromagnetic field into volumes much smaller than that of the incident wavelength. Electrically controllable carrier concentration of graphene opens the door to the use of plasmonic nanoantenna structures with graphene so that the resonant nature of nanoantennas can be tuned. In this study, we demonstrated with the Fourier transform infrared (FTIR) spectroscopy and the Finite Difference Time Domain (FDTD) method that the intensity and resonance peak of bowtie nanoantennas on monolayer graphene can be tuned at mid-infrared (MIR) wavelength regime by applying a gate voltage, since the optical properties of graphene change by changing the carrier concentration.
\end{abstract}

(C) 2014 Elsevier B.V. All rights reserved.

Keywords: Graphene; Nanoantennas; Plasmonics; Bowtie antennas

\section{Introduction}

Graphene is a monolayer of carbon atoms that are arranged in a two-dimensional (2D) honeycomb lattice. Property of the ballistic transport of electrons in graphene makes it a very unique material, since the graphene mobility can reach up to $100,000 \mathrm{~cm}^{2} \mathrm{~V}^{-1} \mathrm{~s}^{-1}$ [1-3]. Electrons in a graphene monolayer, which can travel for micrometers without scattering at room temperature [4], behave as massless Dirac fermions,

\footnotetext{
* Corresponding author at: Nanotechnology Research Center, Bilkent University, Bilkent University, 06800 Ankara, Turkey. Tel.: +90 5359831994 .

E-mail addresses: semihc@bilkent.edu.tr, semihckm@gmail.com (S. Cakmakyapan).
}

where principles of quantum electrodynamics can be tested $[5,6]$. Graphene has attracted great attention due to its exceptional optical properties along with its electrical and mechanical properties. Its optical properties make it ideal for terahertz oscillators, and low-noise electronic and optical sensors [7]. It exhibits a saturable absorption constant as a consequence of Pauli blocking [8-10]. Graphene has been a new candidate for future nanophotonics so far with the numerous applications based on metamaterials [11], photodetectors [12-14], photovoltaics [15], and nanoantennas [16,17], where electronics and plasmonics are combined in nanocircuitry [18]. Surface plasmons that are bound to graphene are confined to volumes on the order of $\sim 10^{6}\left(1 / \alpha^{3}\right)$ times smaller than the diffraction limit, where $\alpha=e^{2} / \hbar c$ is the fine structure constant [19]. As a 
result, these strong light-matter interactions make graphene favorable for new plasmonic applications due to the field enhancement and confinement. The optical conductivity of graphene depends on the doping of the graphene layer at the infrared frequency regime. Since high frequency interband transitions in graphene can be exploited through electrical gating [20], the plasmon resonance of graphene-hybrid structures such as nanoantennas can be modulated by applying a gate voltage $[16,17,21,22]$.

Bowtie antennas are similar to dipole antennas, however they have a larger bandwidth compared to dipole antennas. The broadband nature of bowtie antennas provides flexibility in terms of operation frequency. Bowtie nanoantennas made of gold are critical in applications such as the enhancement of single-molecule fluorescence [23], single-particle trapping [24], and efficient surface-enhanced Raman spectroscopy (SERS) substrate [25], due to strong electromagnetic field enhancement. In this study, the resonance properties of the bowtie nanoantennas at the mid-infrared wavelength regime are controlled by the electrical gating of the graphene layer, where we obtained $113 \mathrm{~nm}$ shift at the resonance peak. It was shown in the earlier study by Emani et al. [17] that the tuning is possible, but limited, by using connected bowtie antennas. Here, the tuning range is further enhanced by using bowtie antennas with a gap, and also by designing the antennas which operate at mid-infrared wavelengths.

\section{Methods}

Commercially available (Graphene Supermarket) monolayer graphene samples grown via Chemical Vapor Deposition (CVD) on p-doped silicon substrates coated with $285 \mathrm{~nm} \mathrm{SiO}_{2}$ dielectric film are used in fabrications. In order to measure the mobility and carrier concentration of graphene samples, Hall measurement, which is a technique to measure the mobility with the help of charge carriers, is performed by fabricating four-contact Van der Pauw devices as shown in Fig. 1(a). The average of several Hall measurements at room temperature from different Van der Pauw devices of a chip sized $1 \mathrm{~cm} \times 1 \mathrm{~cm}$ resulted that the graphene samples are very uniform, and they have a mobility of $2286 \mathrm{~cm}^{2} / \mathrm{V} \mathrm{s}$, and $7.85 \times 10^{12} \mathrm{~cm}^{-2}$ sheet carrier concentration, where the positive carrier concentration means that the majority carriers are holes. In addition, the sheet resistance of the graphene samples is on the order of $1000 \Omega / \square$, which shows a good agreement with the values in the literature [26].

For the preparation of the transistor-like devices with bowtie antennas, we fabricated our own optical mask by using electron beam lithography. The first step of the device fabrication is the ohmic contacts with optical lithography for the drain and source. $20 \mathrm{~nm}$ titanium and $80 \mathrm{~nm}$ gold are deposited as ohmic contact metallization with electron beam evaporator. Then, optical lithography for mesa etching is carried out in order to obtain the active area that connects the source
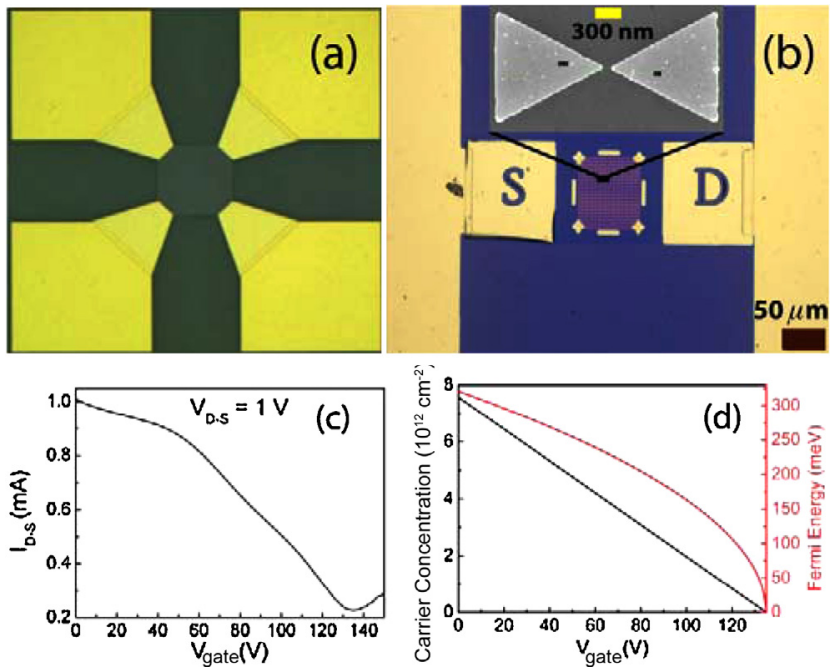

Fig. 1. (a) Fabricated four-contact Van der Pauw device for Hall measurements, (b) optical microscopy image of the tunable bowtie device, i.e. active graphene region with nanostructures between drain (D) and source (S) contacts; inset showing SEM picture of a bowtie antenna, (c) measured drain-source current with respect to the gate voltage, and (d) calculated variation of carrier concentration and Fermi energy with respect to the gate voltage difference $\Delta V$. 
(S) and drain (D), and thus removing the unwanted graphene on the chip. The etching process is carried out with ICP-RIE by exposing samples to $\mathrm{O}_{2}$ plasma for $20 \mathrm{~s}$ with $50 \mathrm{~W}$ RF power, and $1100 \mathrm{~W}$ ICP source power. After that, another optical lithography for interconnect metallization is done in order to be used for probing and bonding purposes. $20 \mathrm{~nm}$ titanium and $200 \mathrm{~nm}$ gold are evaporated as interconnect metals. As the last step of the nanofabrication, bowtie arrays are lithographed on the active region by using electron beam lithography, followed by $5 \mathrm{~nm} \mathrm{Ti}$ and $40 \mathrm{~nm} \mathrm{Au}$ evaporation. Optical microscopy image of a completed fabrication of a device is shown in Fig. 1(b). Scanning electron microscopy (SEM) image of a unit cell of a bowtie antenna array, which will be referred as Sample 1, is given in the inset of Fig. 1(b). Here the gap distance is measured to be $177 \mathrm{~nm}$, total length of a single bowtie is $4.46 \mu \mathrm{m}$, and the flare angle is $58^{\circ}$. Before the optical measurements, dark current-voltage measurements were performed. Charge neutrality point of the graphene sheet is observed to be at $140 \mathrm{~V}$, as plotted in Fig. 1(c). High carrier concentration of graphene, as measured using Van der Pauw devices, results in a large value of charge neutrality point. The reason is predicted to be due to the resist residues on the graphene surface as discussed in Ref. [27].

Theoretical calculations, in order to obtain the Fermi energy dependent refractive index of graphene, are carried out by using the optical conductivity of graphene from the random-phase approximation method $[28,29]$.

$E_{F}=\hbar v_{F} \sqrt{\pi N_{S}}$

Fermi energy, $E_{F}$, can be expressed as in Eq. (1) [30], where $v_{F}$ is the Fermi velocity, and $N_{s}$ is the sheet carrier concentration. The optical conductivity of graphene $[28,29]$ can be changed with different Fermi energies, which is a function of the sheet carrier concentration of graphene, as seen in Eq. (1). It was demonstrated in Refs. [30,31] that the charge concentration is linearly proportional with the applied gate voltage and, therefore, the optical conductivity of graphene can be tuned at different gate voltages. Relation between the applied gate voltage and carrier concentration/Fermi energy is plotted in Fig. 1(d). Here the voltage difference, $\Delta V$, is defined by $\Delta V=\left|V_{g}-V_{\mathrm{CNP}}\right|$, where $V_{g}$ is the gate voltage and $V_{\mathrm{CNP}}$ the voltage at the charge neutrality point.

\section{Results and discussion}

Fourier transform infrared (FTIR) spectroscopy measurement results for Sample 1 are given in Fig. 2. Reflection spectrum in Fig. 2(a) shows that reflectivity changes with a blue shift, as the voltage difference increases, since the refractive index of the graphene layer changes with the applied voltage. There is a $113 \mathrm{~nm}$ shift at the peaks between $\Delta V=0 \mathrm{~V}$ and $140 \mathrm{~V}$ cases, as demonstrated in the inset of Fig. 2(a). Along with the blue shift, reflection curves exhibit an asymmetric nature with the applied voltage. The reason of this asymmetry is that the tuning of the curves are much more pronounced at longer wavelengths, because the optical conductivity of graphene changes more at the longer wavelengths for different Fermi energies. In other words, graphene is more sensitive at longer wavelengths. It is also observed that the resonant curves narrow down for larger $\Delta V$, which will be discussed later. Relative reflectivity with respect to the reflection at the charge neutrality point is shown in Fig. 2(b). It is seen that the curves blue shift by applied voltage.
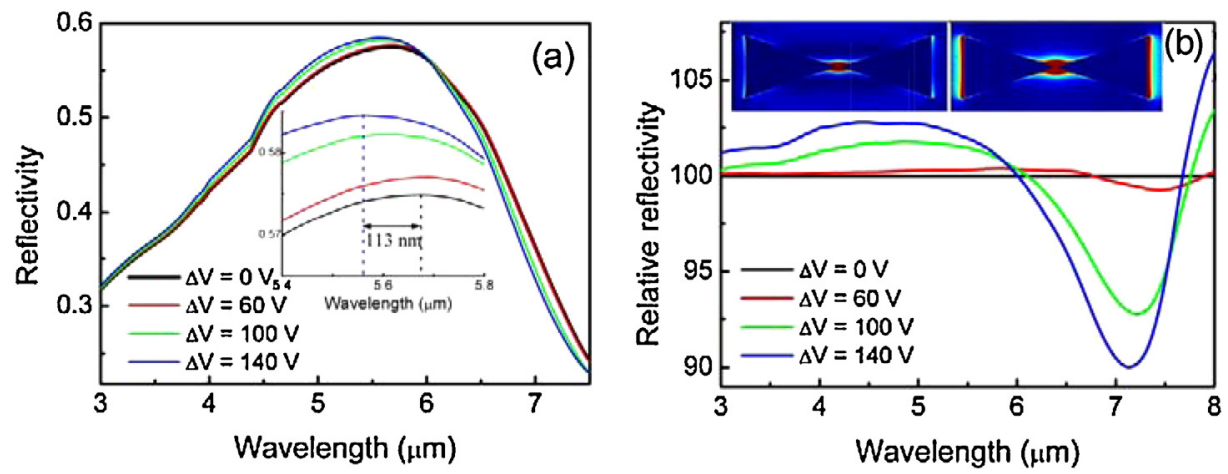

Fig. 2. (a) Measured reflectance spectra at different $\Delta V$; enlarged view of resonance peaks shown in the inset, and (b) relative reflectivity of bowtie antenna array at different gate voltages; inset showing the $x$-component of electric field intensity for Sample 1 at $5.5 \mu \mathrm{m}$ and $7 \mu \mathrm{m}$. (For interpretation of the references to color in text, the reader is referred to the web version of this article.) 
Calculated results of $|E x|^{2}$ on the surface at the wavelengths of $5.5 \mu \mathrm{m}$ and $7.0 \mu \mathrm{m}$ are demonstrated in the inset of Fig. 2(b), in order to be able to understand the reason of the intensity change at $7 \mu \mathrm{m}$. The real and imaginary parts of the refractive index of graphene are calculated for different Fermi energies, and hence different gate voltages. The numerical simulations are performed by using commercially available software package Lumerical by embedding these refractive index data as a new material. Field intensity and localization at off-resonance wavelength $(7 \mu \mathrm{m})$ is higher than that of resonance wavelength $5.5 \mu \mathrm{m}$, where the field is mainly localized at the gap region. Additionally, there is a coupling between neighbor bowties at $7.0 \mu \mathrm{m}$, and the tunability of the curves depends on the interaction volume between light and graphene. Since the total interaction volume is higher at $7.0 \mu \mathrm{m}$, electric field at this wavelength results more interaction with the graphene layer, and the generated $\mathrm{e}-\mathrm{h}$ pairs decrease the resonance more around $7 \mu \mathrm{m}$. Calculated reflectivity of the structures under different bias voltage is also shown in Fig. 3, where the resonance shift is approximately $190 \mathrm{~nm}$. Fermi energy of the graphene is converted to the voltage difference according to the relation given in Fig. 1(d). The results are in agreement with the measurements. The discrepancies are due to the fabrication imperfections, and the possible defects and grain boundaries on graphene surface.

Quality factor and line width difference, $\Delta \lambda$, variation under different gate bias are shown in Fig. 4. Quality factor is the ratio between the resonance peak and the bandwidth of the reflection curve. Line width represents the full width half maximum of a reflectivity curve, and $\Delta \lambda$ is the difference of full width half maximum values relative to the curve at $\Delta V=0$. Line width of the reflectivity peak decreases, as the gate voltage difference, $\Delta V$, increases. In other words, curves narrow down up to $120 \mathrm{~nm}$ at larger $\Delta V$. Therefore, the

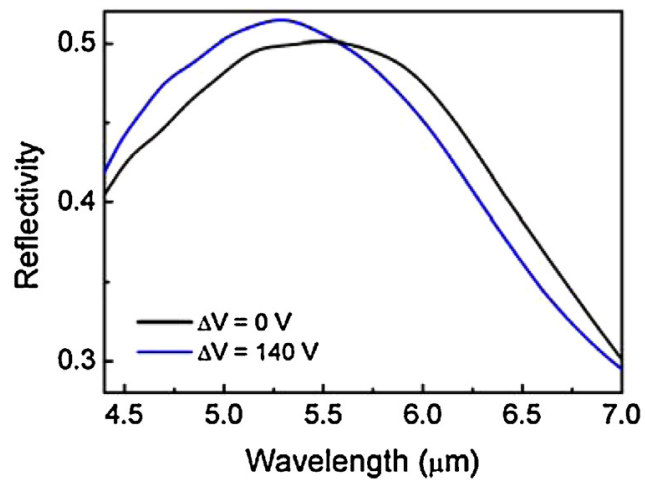

Fig. 3. Calculated reflectance spectra at different $\Delta V$.

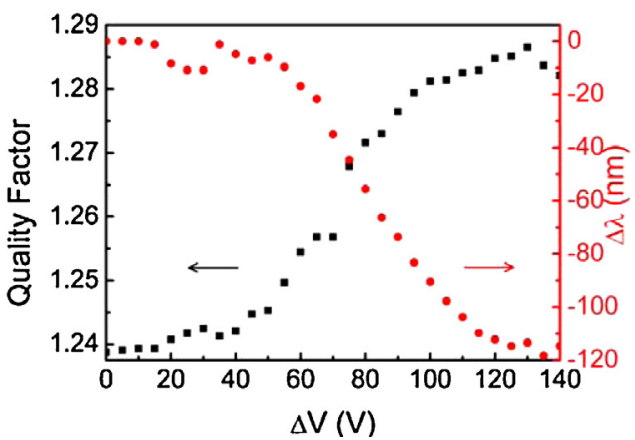

Fig. 4. Quality factor (black squares) and line width difference (red dots) under different gate bias. (For interpretation of the references to color in this figure legend, the reader is referred to the web version of this article.)

quality factor increases. Incident photons having energies higher than $2 E_{F}$ are absorbed, since they can generate electron-hole pairs. At the charge neutrality point, $\Delta V=0$, Fermi energy goes to zero, according to Eq. (1). This results in more interband transitions due to the incident photons, thus increasing the absorption, because almost all of the photons are able to exceed $2 E_{F}$. The results shown in Fig. 4 are consistent with this qualitative explanation. The reflection curve at the charge neutrality point is the broadest one having the lowest quality factor.

The importance of the coupling between the neighboring bowties can be further analyzed. The graphene between the neighboring bowties in $x$-direction is removed in simulation environment. The comparison between two cases, where there is graphene on the entire surface and graphene removed for isolation, is demonstrated in Fig. 5. The sheet concentration of graphene is taken to be $7.85 \times 10^{12} \mathrm{~cm}^{-2}$, which corresponds to

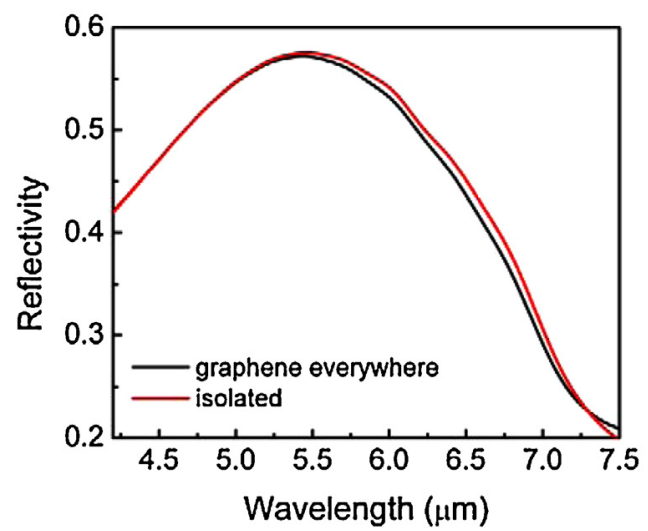

Fig. 5. Comparison of calculated reflectivity between the structure with graphene everywhere and graphene removed between two neighbors in $x$-direction. 

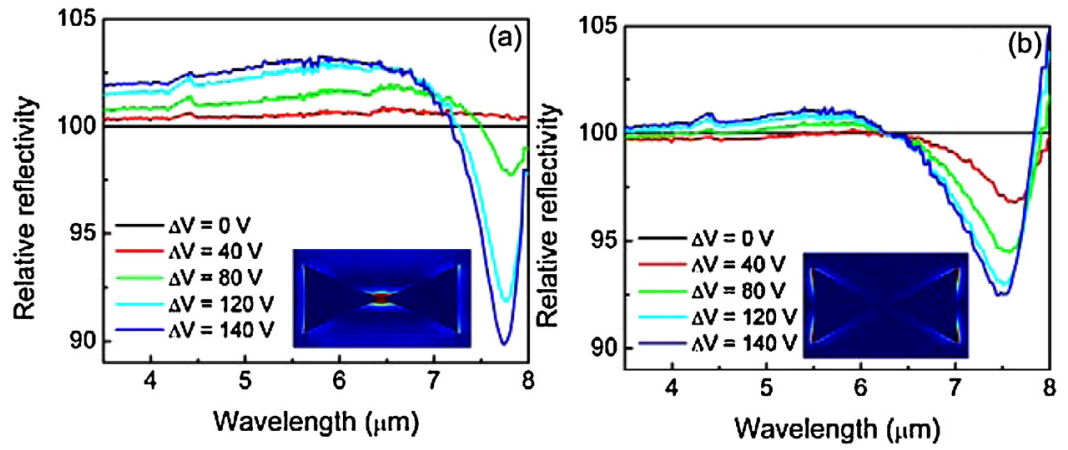

Fig. 6. Relative reflectivity of Sample 2 for (a) TE, electric field in the $x$-direction, and (b) TM polarization.

$\Delta V=140 \mathrm{~V}$ case in our experiments. As seen in the figure, results do not change up to the resonance frequency, however, there is a shift at the larger wavelengths. Therefore, it is important for this case to have graphene everywhere on the surface, because the interactions increase, when there is electric field enhancement, which also increases the tuning capability of the devices.

Experimental results for another bowtie antenna array, Sample 2, that operates at $7 \mu \mathrm{m}$ are given in Fig. 6(a) and (b) for TE and TM polarized incident waves, respectively. The total length of a single bowtie is $6.1 \mu \mathrm{m}$, and the flare angle is $52^{\circ}$. It is observed that the damping effect is higher for the case of TE polarization, where the electric field component of the wave is in the $x$-direction. The excitation of the bowtie gap and the coupling between two neighbor antennas can be provided by TE polarization, whereas TM polarization only excites the edges of the triangles. Namely, it works as a periodic array made of triangles, which can be seen from the field distribution insets of Fig. 6. As a result, the effective graphene area is larger for the case of TE. Therefore, reflectivity is more sensitive to the voltage difference.

\section{Conclusion}

In summary, we demonstrated the resonance tunability of bowtie nanoantennas with the help of electrically gated graphene samples. We obtained a frequency shift, resonance damping, and enhancement by fabricating bowtie structures on a graphene layer. Electrical tuning of antenna resonance with a varying gate bias is studied with a theoretical modeling and confirmed experimentally. As graphene-based devices are already being realized at optical frequencies, it is possible to use these bowtie and graphene-based structures for new optical applications such as novel photovoltaic, ultrafast miniature photodetectors and optical switches. The research for novel applications like optical switches will be conducted by us in the future.

\section{Acknowledgments}

This work is supported by the projects DPT-HAMIT, ESF-EPIGRAT, NATO-SET-181 and TUBITAK under Project Nos., 107A004, 109A015, 109E301. One of the authors (E.O.) also acknowledges partial support from the Turkish Academy of Sciences.

\section{References}

[1] A.K. Geim, K.S. Novoselov, Nat. Mater. 3 (6) (2007) 183-191.

[2] A.H.C. Neto, Abstr. Pap. Am. Chem. Soc. 238 (2009).

[3] X. Du, I. Skachko, E.Y. Andrei, Int. J. Mod. Phys. B 22 (25-26) (2008) 4579-4588.

[4] F.H.L. Koppens, D.E. Chang, F.J.G. de Abajo, Nano Lett. 11 (8) (2011) 3370-3377.

[5] A.C. Neto, F. Guinea, N.M.R. Peres, Phys. World 19 (11) (2006) 33-37.

[6] K.S. Novoselov, E. McCann, S.V. Morozov, V.I. Fal'ko, M.I. Katsnelson, U. Zeitler, D. Jiang, F. Schedin, A.K. Geim, Nat. Phys. 2 (3) (2006) 177-180.

[7] F. Rana, IEEE Trans. Nanotechnol. 7 (1) (2008) 91-99.

[8] F. Bonaccorso, Z. Sun, T. Hasan, A.C. Ferrari, Nat. Photon. 4 (9) (2010) 611-622.

[9] T. Hasan, Z.P. Sun, F.Q. Wang, F. Bonaccorso, P.H. Tan, A.G. Rozhin, A.C. Ferrari, Adv. Mater. 21 (38-39) (2009) 3874-3899.

[10] Z.P. Sun, T. Hasan, F. Torrisi, D. Popa, G. Privitera, F.Q. Wang, F. Bonaccorso, D.M. Basko, A.C. Ferrari, ACS Nano 4 (2) (2010) 803-810.

[11] N. Papasimakis, Z.Q. Luo, Z.X. Shen, F. De Angelis, E. Di Fabrizio, A.E. Nikolaenko, N.I. Zheludev, Opt. Express 18 (8) (2010) 8353-8359

[12] Q. Gao, J. Guo, J. Appl. Phys. 112 (8) (2012).

[13] T. Mueller, F.N.A. Xia, P. Avouris, Nat. Photon. 4 (5) (2010) 297-301.

[14] Z.Y. Fang, Z. Liu, Y.M. Wang, P.M. Ajayan, P. Nordlander, N.J. Halas, Nano Lett. 12 (7) (2012) 3808-3813. 
[15] M. Freitag, T. Low, F.N. Xia, P. Avouris, Nat. Photon. 7 (1) (2013) 53-59.

[16] Y. Yao, M.A. Kats, P. Genevet, N. Yu, Y. Song, J. Kong, F. Capasso, Nano Lett. 13 (3) (2013) 1257-1264.

[17] N.K. Emani, T.F. Chung, X. Ni, A.V. Kildishev, Y.P. Chen, A. Boltasseva, Nano Lett. 12 (10) (2012) 5202-5206.

[18] E. Ozbay, Science 311 (5758) (2006) 189-193.

[19] J.N. Chen, M. Badioli, P. Alonso-Gonzalez, S. Thongrattanasiri, F. Huth, J. Osmond, M. Spasenovic, A. Centeno, A. Pesquera, P. Godignon, A.Z. Elorza, N. Camara, F.J.G. de Abajo, R. Hillenbrand, F.H.L. Koppens, Nature 487 (7405) (2012) 77-81.

[20] R.R. Nair, P. Blake, A.N. Grigorenko, K.S. Novoselov, T.J. Booth, T. Stauber, N.M.R. Peres, A.K. Geim, Science 320 (5881) (2008) 1308

[21] L. Ju, B.S. Geng, J. Horng, C. Girit, M. Martin, Z. Hao, H.A. Bechtel, X.G. Liang, A. Zettl, Y.R. Shen, F. Wang, Nat. Nanotechnol. 6 (10) (2011) 630-634.

[22] S. Cakmakyapan, L. Sahin, F. Pierini, W. Strupinski, E. Ozbay, Appl. Phys. Lett. 103 (18) (2013).
[23] A. Kinkhabwala, Z.F. Yu, S.H. Fan, Y. Avlasevich, K. Mullen, W.E. Moerner, Nat. Photon. 3 (11) (2009) 654-657.

[24] B.J. Roxworthy, K.D. Ko, A. Kumar, K.H. Fung, E.K. Chow, G.L. Liu, N.X. Fang, K.C. Toussaint Jr., Nano Lett. 12 (2) (2012) 796-801.

[25] N.A. Hatab, C.H. Hsueh, A.L. Gaddis, S.T. Retterer, J.H. Li, G. Eres, Z. Zhang, B. Gu, Nano Lett. 10 (2010) 4952-4955.

[26] P. Tassin, T. Koschny, C.M. Soukoulis, Science 341 (6146) (2013) 620-621.

[27] Z. Cheng, Q. Zhou, C. Wang, Q. Li, Y. Fang, Nano Lett. 11 (2) (2011) 767-771.

[28] L.A. Falkovsky, S.S. Pershoguba, Phys. Rev. B 76 (15) (2007).

[29] L.A. Falkovsky, A.A. Varlamov, Eur. Phys. J. B 56 (4) (2007) 281-284.

[30] K.S. Novoselov, A.K. Geim, S.V. Morozov, D. Jiang, M.I. Katsnelson, I.V. Grigorieva, S.V. Dubonos, A.A. Firsov, Nature 438 (7065) (2005) 197-200.

[31] Z.Q. Li, E.A. Henriksen, Z. Jiang, Z. Hao, M.C. Martin, P. Kim, H.L. Stormer, D.N. Basov, Nat. Phys. 4 (7) (2008) 532-535. 